\title{
O uso da L-carnitina como adjuvante no tratamento da miocardiopatia dilatada em criança com Aids
}

\author{
Usage of L-carnitine as adjuvant in the treatment of dilated cardiomyopathy in a child with Aids
}

Lourdes Zélia Zanoni ${ }^{1}$, Ana Lúcia L. Oliveira², Luiz Carlos T. Cônsolo ${ }^{3}$, Carlos Eduardo Z. Cônsolo ${ }^{4}$ Yara Delamare Espíndola ${ }^{4}$

\section{RESUMO}

Objetivo: Apresentar a resposta cardiovascular à L-carnitina de um paciente com insuficiência cardíaca congestiva decorrente de miocardiopatia dilatada pelo vírus da imunodeficiência humana.

Descrição do caso: Criança com quadro clínico de insuficiência cardíaca congestiva grave devido à miocardiopatia dilatada pela síndrome de imunodeficiência adquirida. $\mathrm{O}$ tratamento para as manifestações clínicas foi instituído, com pouca resposta clínica. Com objetivo de melhorar o desempenho energético/ metabólico dos cardiomiócitos, foi instituída terapia com Lcarnitina. Observou-se significativa melhora clínica do paciente, em relação ao desempenho cardíaco, mesmo antes do início do tratamento com os fármacos antirretrovirais.

Comentários: A L-carnitina é um composto que facilita o transporte dos ácidos graxos de cadeia longa para dentro da mitocôndria. Nesse caso, o uso da L-carnitina parece ser clinica e bioquimicamente justificado.

Palavras-chave: L-carnitina; cardiomiopatia dilatada; síndrome de imunodeficiência adquirida; criança.

\section{ABSTRACT}

Objective: To present the cardiovascular response to Lcarnitine of a patient with congestive heart failure caused by dilated cardiomyopathy and human immunodeficiency virus.

Case description: Child with a clinical history of severe congestive heart failure due to dilated cardiomyopathy caused by acquired immunodeficiency syndrome. The treatment for the symptoms resulted in a poor clinical response. In order to improve the energetic performance/metabolism of cardiomyocytes, therapy with L-carnitine was established. There was significant clinical improvement of the cardiac performance of the patient, even before starting the treatment with antiretroviral drugs.

Comments: L-carnitine is a compound that facilitates the transport of long-chain fatty acids into the mitochondria. In this case the administration of L-carnitine appears to be clinically and biochemical justified.

Key-words: L-carnitine; cardiomyopathy, dilated; acquired immunodeficiency syndrome; child.
Instituição: Universidade Federal de Mato Grosso do Sul (UFMS), Campo Grande, MS, Brasil

'Doutora em Ciências da Saúde pela UFMS; Professora do Departamento de Pediatria da UFMS, Campo Grande, MS, Brasil

${ }^{2}$ Doutora em Medicina Tropical pelo Instituto Oswaldo Cruz (Fiocruz), Professora do Departamento de Pediatria da UFMS, Campo Grande, MS, Brasil ${ }^{3}$ Mestre em Ciências da Saúde pela UFMS; Professor do Departamento de Pediatria da UFMS, Campo Grande, MS, Brasil

${ }^{4}$ Acadêmico do Curso de Medicina da Universidade Anhanguera-Uniderp, Campo Grande, MS, Brasil
Endereço para correspondência:

Lourdes Zélia Zanoni

Rua Alexandre, 378 - Giocondo Orsi

CEP 79022-080 - Campo Grande/MS

E-mail: Izzanoni@terra.com.br

Conflito de interesse: nada a declarar

Recebido em: 20/12/2009

Aprovado em: 30/6/2010 


\section{Introdução}

A infecção pelo vírus da imunodeficiência humana (HIV) constitui um dos maiores problemas de Saúde Pública mundial, com alta mortalidade e morbidade, atingindo pessoas jovens em plena fase produtiva e de reprodução.

No Brasil, acompanhando a tendência mundial, houve crescimento progressivo do número de casos em mulheres jovens, bem como da transmissão perinatal, que responde hoje por mais de $80 \%$ do total dos casos de síndrome de imunodeficiência adquirida (Aids) pediátricos notificados ${ }^{(1)}$. As crianças acometidas por infecção vertical têm um período clínico de latência menor e a progressão da doença é mais rápida ${ }^{(2)}$.

O comprometimento cardíaco é decorrente da agressão causada pelo próprio HIV, além das infecções oportunistas, cardiotoxicidade causada pelas drogas antirretrovirais, deficiência nutricional e imunossupressão prolongada ${ }^{(1,3-6)}$. As principais manifestações cardíacas são miocardiopatia dilatada, derrame pericárdico, doença coronariana e insuficiência cardíaca congestiva (ICC) $)^{(4,7,8)}$.

A L-carnitina é um composto que, na mitocôndria, facilita o transporte dos ácidos graxos de cadeia longa que entram no ciclo da betaoxidação e são convertidos em acetilcoenzima A, para posterior entrada no ciclo de Krebs e na cadeia respiratória, com produção de energia ${ }^{(9-11)}$.

A levocarnitina também possui ações cardioprotetoras frente à hipóxia e ao estresse oxidativo, diminuindo a produção de radicais livres e o consequente dano mitocondrial, melhorando a reparação das membranas celulares lesadas, reduzindo o processo de apoptose e influenciando no remodelamento cardíaco ${ }^{(8,12,13)}$.

O tratamento da cardiomiopatia dilatada secundária aos defeitos da betaoxidação tem como pedra angular a suplementação de L-carnitina, mas também são demonstrados efeitos positivos nos sintomas dos pacientes com ICC de outras etiologias ${ }^{(9,14)}$.

O presente artigo teve como objetivo apresentar a resposta cardiovascular à L-carnitina de um paciente com miocardiopatia dilatada pelo HIV e ICC.

\section{Descrição do caso}

Criança branca do sexo masculino, 3,8 anos de idade, foi atendida no Centro de Terapia Intensiva (CTI) do Hospital Universitário da Universidade Federal de Mato Grosso do Sul (UFMS), em Campo Grande (MS), com hipótese diagnóstica de miocardiopatia dilatada, pneumonia e desnutrição grave.
A mãe do paciente relatou que, há cerca de um ano e quatro meses, a criança vinha apresentando dispneia progressiva aos esforços e cianose perioral. Procurou assistência médica sem melhora do quadro. Há três meses, mostrava aumento progressivo do volume abdominal. Há 18 dias, iniciou dispneia em repouso. Ao exame clínico, apresentava-se em mau estado geral, desnutrido, consciente, irritado, hipocorado, hidratado, com frequência cardíaca de 184 batimentos/minuto, frequência respiratória de 55 incursões/minuto, pressão arterial de $126 / 74 \mathrm{mmHg}$, peso $12,2 \mathrm{~kg}(<$ percentil 3$)$, temperatura axilar de $39,2^{\circ} \mathrm{C}$ e saturação de $\mathrm{O}_{2}$ de $90 \%$. Otoscopia, oroscopia e rinoscopia eram normais. Os gânglios eram pequenos, indolores, móveis e não aderentes a planos profundos, sendo palpáveis nas regiõos cervical, inguinal e submandibular. A ausculta cardíaca indicava bulhas taquicárdicas, em ritmo de galope, sem sopros. Quanto ao aparelho respiratório, notava-se tiragem intercostal e ausculta pulmonar com raros estertores crepitantes bilaterais. O abdome era globoso, flácido, com fígado palpável a $10 \mathrm{~cm}$ do rebordo costal direito doloroso e baço palpável a $7 \mathrm{~cm}$ do rebordo costal esquerdo. O paciente apresentava perfusão periférica lentificada e cianose de extremidades. Os exames laboratoriais evidenciaram anemia (hemoglobina: 10,4g\%; hematócrito: 33\%); leucocitose $\left(17.000 \mathrm{~mm}^{3}\right) \mathrm{com}$ neutrofilia (59\%); ureia: $47 \mathrm{mg} / \mathrm{dL}$; creatinina: $0,3 \mathrm{mg} / \mathrm{dL}$, AST: 39U/L; ALT: 28U/L; eletrólitos normais (Na: 137mmol/L; K: 5,3mmol/L; Ca: 8,9mg/dL; P: 3,1mg/dL; $\mathrm{Mg}: 2,2 \mathrm{mg} / \mathrm{dL})$; e hipoalbuminemia $(2,7 \mathrm{~g} / \mathrm{dL})$. Ao raio $\mathrm{X}$ de tórax, notou-se opacidade alvéolo/intersticial na região peri-hilar, e observaram-se base dos pulmões e cardiomegalia com índice cardiotorácico de 65\% (Figura 1). O eletrocardiograma apresentou ritmo sinusal e alteração difusa de repolarização ventricular. O ecocardiograma evidenciou fração de ejeção de 0,41 e encurtamento sistólico

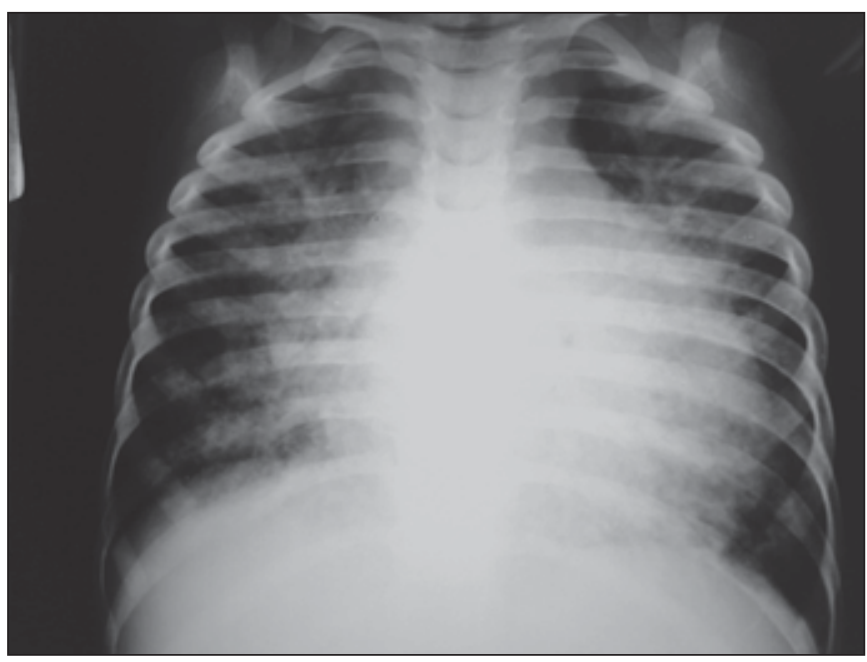

Figura 1 - Evidente cardiomegalia e sinais de congestão circulatória pulmonar. 
de 19,9\%, comprometimento miocárdico difuso e importante do ventrículo esquerdo, disfunção diastólica leve, hipocinesia difusa e derrame pericárdico discreto (Figura 2). A hemocultura foi positiva para Candida sp. As sorologias eram negativas para citomegalovírus, mononucleose e toxoplasmose. À internação colheu-se sorologia para HIV, embora na anamnese não houvesse nenhum antecedente sugestivo de doença pelo HIV.

O paciente permaneceu internado durante 15 dias, com antibioticoterapia, antifúngico e tratamento para ICC. Apresentou discreta melhora do quadro clínico, mantendo dispneia aos mínimos esforços e hepatomegalia. A prescrição de inotrópico, vasodilatador (inibidor da enzima conversora de angiotensina), diurético e betabloqueador foi mantida e iniciou-se a administração de L-carnitina, na dose de 100 $\mathrm{mg} / \mathrm{kg} / \mathrm{dia}$, por via oral, dividida em duas doses. Após a alta, o paciente foi controlado no ambulatório de cardiopediatria semanalmente quanto ao quadro clínico e ao uso correto das medicações.

Após cerca de 45 dias de evolução, foi observada melhora da sintomatologia, com diminuição significativa da

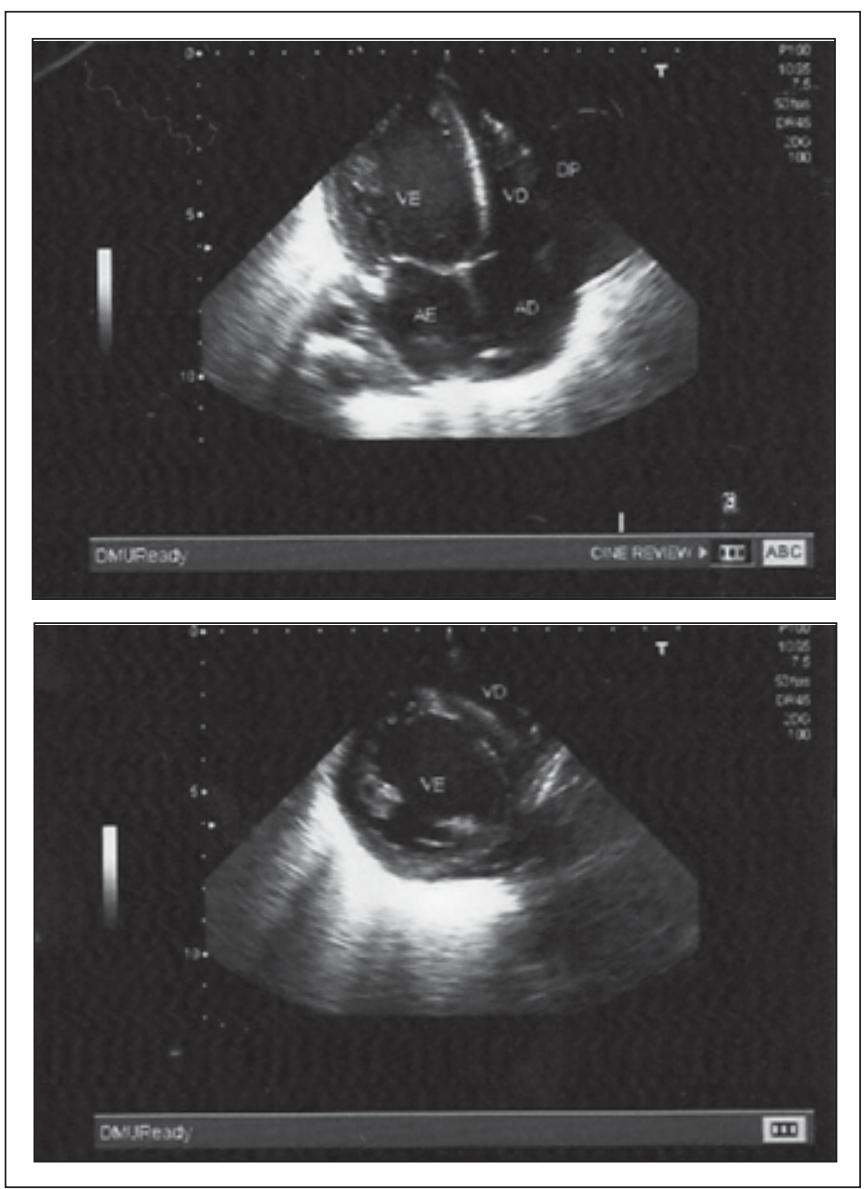

Figura 2 - Ecocardiograma evidenciando comprometimento miocárdico de ventrículo esquerdo. dispneia e ganho de peso de 1000g. Ao exame clínico, a criança persistia com taquipneia, tiragem intercostal, porém com ausculta normal, hepatomegalia não dolorosa de $8 \mathrm{~cm}$, esplenomegalia de $6 \mathrm{~cm}$ e ausência de edema. O raio $\mathrm{X}$ de tórax evidenciou diminuição da área cardíaca e imagens pulmonares inalteradas, diagnosticada como pneumonia intersticial linfocítica (Figura 3). Novo ecocardiograma mostrou fração de ejeção de 0,67 e encurtamento sistólico de 36\% (Figura 4). Nessa ocasião, o resultado da sorologia para o HIV foi obtido, sendo positivo. A contagem de linfócitos $\mathrm{CD}^{+}$era de 198 células $/ \mathrm{mm}^{3}$, sendo iniciado o tratamento específico para Aids com zidovudina, lamivudina e efavirenz, além de corticoterapia (prednisona: $2 \mathrm{mg} / \mathrm{kg} / \mathrm{dia}$ ) e quimioprofilaxia para Pneumocistis carini (sulfametoxazol e trimetropina: $20 \mathrm{mg} / \mathrm{kg} /$ dia, baseada na sulfa).

\section{Discussão}

A Aids é uma doença sistêmica, infectocontagiosa, caracterizada por imunossupressão, que predispõe os pacientes às infecções oportunistas e neoplasias. É de natureza progressiva, podendo comprometer o aparelho cardiovascular de múltiplas formas. A prevalência das anormalidades cardíacas parece estar subestimada, tanto na população adulta como na infantil, com valores entre 28 e $73 \%{ }^{(3,15)}$.

$\mathrm{O}$ acometimento cardíaco na criança infectada pelo HIV-1 ocorre em torno de $50 \%$ dos casos, após aproximadamente 18 meses. As manifestações clínicas mais comuns são a miocardiopatia dilatada e a insuficiência cardíaca - em

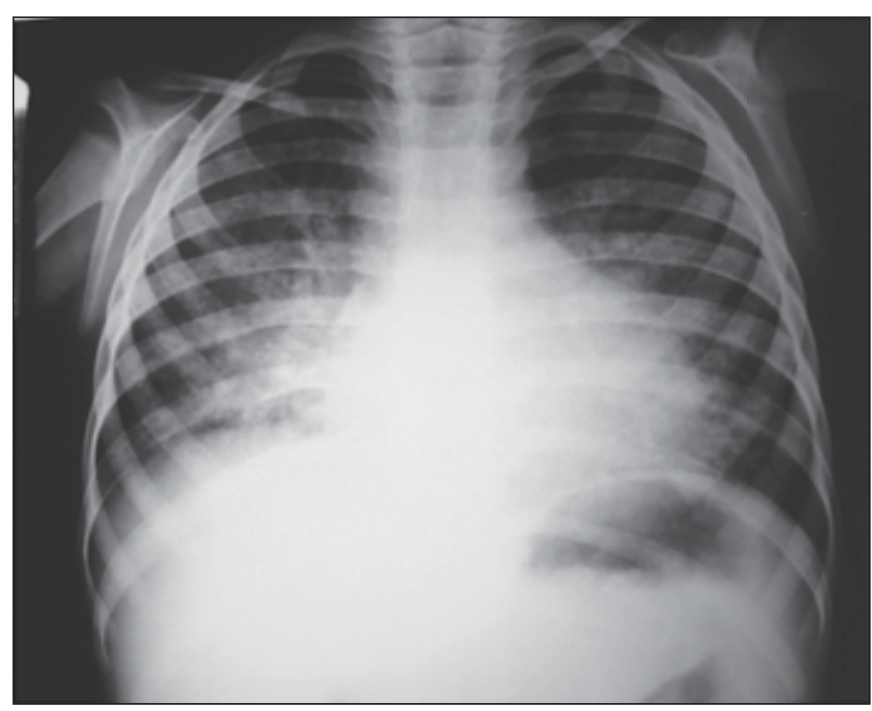

Figura 3 - Redução significativa da área cardíaca. Infiltrado reticulonodular bilateral predominante em bases pulmonares. 

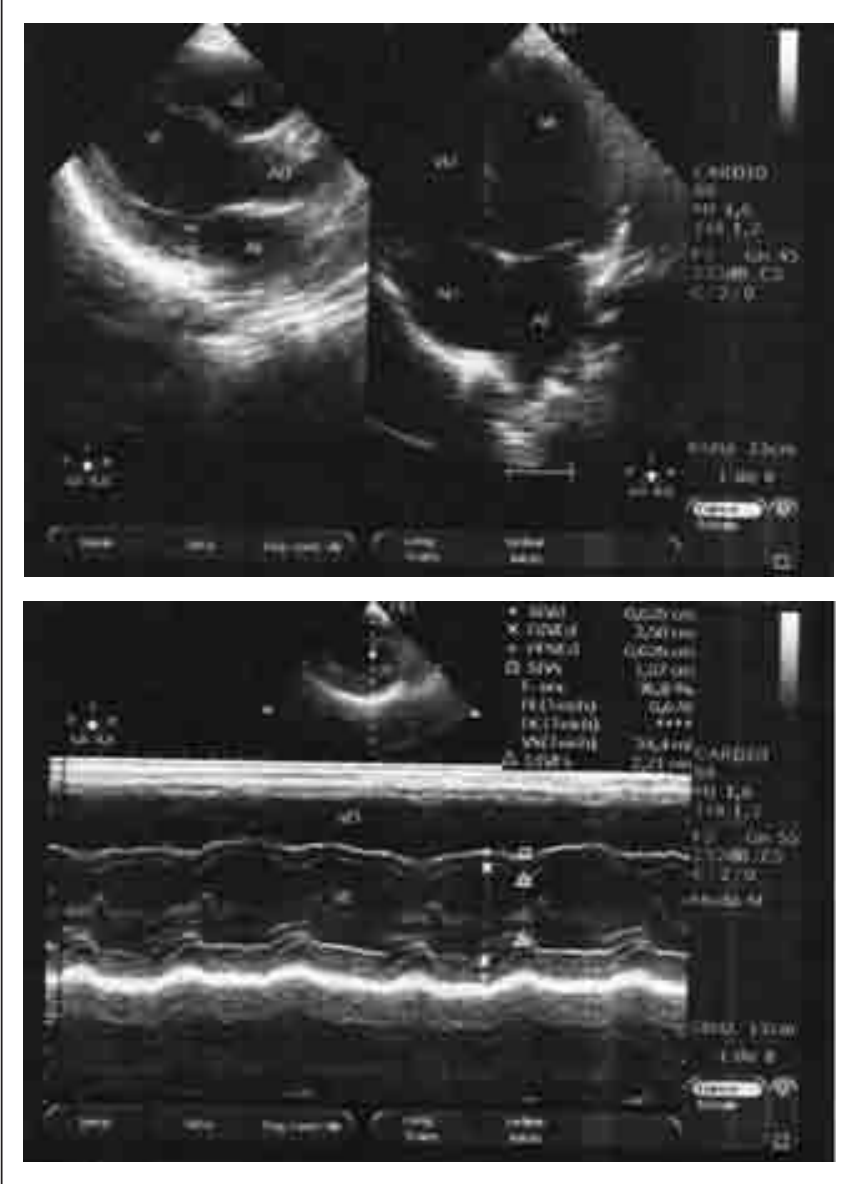

Figura 4 - Ecocardiograma evidenciando melhora da função em relação ao exame anterior.

torno de $12 \%^{(3,4,16)}$. Mesmo em pacientes assintomáticos do ponto de vista cardiovascular, $50 \%$ apresentam miocardite linfocítica intersticial nos exames anatomopatológicos. Esse número eleva-se para $83 \%$ naqueles que evoluem com ICC. O mecanismo pelo qual o coração é lesado na Aids ainda não está elucidado. A terapia antirretroviral modificou substancialmente o curso da miocardiopatia dilatada causada pelo HIV, aumentando a sobrevida ${ }^{(17)}$. Registra-se mortalidade na infância por complicações cardíacas em torno de 1 a $9 \%{ }^{(4,18,19)}$.

Neste caso, conforme relatado na literatura, o comprometimento cardíaco também ocorreu por volta dos dois anos de idade. Dada a gravidade do caso aqui relatado, para melhorar o desempenho energético/metabólico dos cardiomiócitos remanescentes, ativando o metabolismo miocárdico e como um coadjuvante à terapia para ICC, decidiu-se utilizar a L-carnitina $(100 \mathrm{mg} / \mathrm{kg} / \mathrm{dia})^{(9,12)}$. Em pacientes adultos portadores de cardiomiopatia dilatada, que receberam suplementação de L-carnitina na dose de $2 \mathrm{~g} /$ dia, foi demonstrada maior sobrevida em três anos em relação ao grupo controle ${ }^{(20)}$. Também na insuficiência cardíaca moderada a grave, essa substância aumentou a tolerância aos exercícios ${ }^{(14)}$.

A melhora clínica do ponto de vista cardiovascular, observada neste caso, ocorreu de forma evidente e substancial após o início da L-carnitina e antes do início da terapia antirretroviral, tendo em vista que as medicações para ICC já estavam previamente em uso, em doses adequadas, e não se constatava melhora do paciente. Em pacientes com miocardiopatia dilatada, foram demonstrados efeitos benéficos da reposição da L-carnitina, havendo aumento da fração de ejeção ao ecocardiograma e melhor desempenho energético/ metabólico dos cardiomiócitos remanescentes, com maior geração de força muscular por unidade contrátil, responsável pela melhora da função cardíaca ${ }^{(12)}$.

Considerando a relevância e a presença cada vez maior no nosso meio de pais portadores de Aids, a investigação clínica e laboratorial dessa doença deve fazer parte do atendimento a toda criança com miocardiopatia dilatada e ICC. Evidenciouse, neste caso, a possibilidade de melhora da função cardíaca com o uso de L-carnitina como um adjuvante ao tratamento antirretroviral na criança com Aids. Entretanto, outros estudos são necessários para comprovar o papel de tal substância na criança com miocardiopatia pelo HIV.

\section{Referências bibliográficas}

1. Cunha MC, Siqueira-Filho AG, Santos SR, Abreu TF, Oliveira RH, Baptista DM et al. AIDS na infância: acometimento cardíaco com e sem a terapia antiretroviral tríplice combinada. Arq Bras Cardiol 2008;90:11-7.

2. Shearer WT, Lipshultz SE, Easley KA, Mclntosh K, Pitt J, Quinn TC et al. Alterations in cardiac and pulmonary function in pediatric rapid human immunodeficiency virus type 1 disease progressors. Pediatric Pulmonary and Cardiovascular Complications of Vertically Transmitted Human Immunodeficiency Virus Study Group. Pediatrics 2000;105:e9.

3. Diógenes MS, Succi RC, Machado DM, Moisés VA, Novo NF, Carvalho

AC. Estudo cardiológico longitudinal em crianças expostas ao vírus da imunodeficiência humana tipo 1 por via perinatal. Arq Bras Cardiol 2005;85:233-40.

4. Hajjar LA, Calderaro D, Yu PC, Giuliano I, Lima EM, Barbaro G et al. Cardiovascular manifestations in patients infected with the human immunodeficiency virus. Arq Bras Cardiol 2005;85:363-77.

5. Shah I, Prabhu SS, Sumitra V, Shashikiran HS. Cardiac dysfunction in HIV infected children: a pilot study. Indian Pediatr 2005;42:146-9.

6. Barbaro G, Di Lorenzo G, Grisorio B, Barbarini G. Incidence of dilated 
cardiomyopathy and detection of HIV in myocardial cells of HIV-positive patients. N Engl J Med 1998;339:1093-9.

7. Barbarinia $G$, Barbaro $G$. Incidence of the involvement of the cardiovascular system in HIV infection. AIDS. 2003;17 (Suppl 1):S46-50.

8. Prendergast BD. HIV and cardiovascular medicine. Heart 2003;89:793-800.

9. Helton E, Darragh R, Francis P, Fricker FJ, Jue K, Koch G et al. Metabolic aspects of myocardial disease and a role of L-carnitine in the treatment of childhood cardiomyopathy. Pediatrics 2000;105:1260-70.

10. Winter SC, Buist NR. Cardiomyopathy in childhood, mitochondrial dysfunction, and the role of L-carnitine. Am Heart J 2000;139:S63-9.

11. Winter S, Jue K, Prochazka J, Francis P, Hamilton W, Linn L et al. The role of L-carnitine in pediatric cardiomyopathy. J Child Neurol 1995;10 (Suppl 2):S45-51.

12. Azevedo VM, Albanesi-Filho FM, Santos MA, Castier MB, Cunha MO. The role of L-carnitine in nutritional status and echocardiographic parameters in idiopathic dilated cardiomyopathy in children. J Pediatr (Rio J) 2005; 81:368-72.

13. Pierpont ME, Breningstall GN, Stanley CA, Singh A. Familial carnitine transporter defect: a treatable cause of cardiomyopathy in children. Am Heart J 2000;139:S96-106.

14. Pauly DF, Pepine CJ. The role of carnitine in myocardial dysfunction. Am J Kidney Dis. 2003;41 (Suppl 4):S35-43.
15. Rerkpattanapipat P, Wongpraparut N, Jacobs LE, Kotler MN. Cardiac manifestations of acquired immunodeficiency syndrome. Arch Intern Med 2000; $160: 602-8$

16. Rizos I. Three-year survival of patients with heart failure caused by dilated cardiomyopathy and L-carnitine administration. Am Heart $\mathrm{J}$ 2000;139:S120-3.

17. Sani MU. Myocardial disease in human immunodeficiency virus (HIV) infection: a review. Wien Klin Wochenschr 2008;120:77-87.

18. Lipshultz SE, Easley KA, Orav EJ, Kaplan S, Starc TJ, Bricker JT et al. Left ventricular structure and function in children infected with human immnodeficiency virus - The Prospective $\mathrm{P}^{2} \mathrm{C}^{2}$ HIV Multicenter Study. Circulation 1998;97:1246-56.

19. Tovo RA, de Martino M, Gabiano C, Cappello N, D'Elia R, Loy A et al Prognostic factors and survival in children with perinatal HIV-1 infection. The Italian Register for HIV Infections in Children. Lancet 1992;339: 1249-53.

20. Starc TJ, Lipshultz SE, Kaplan S, Easley KA, Bricker JT, Colan SD et al Cardiac complications in children with human immunodeficiency virus infection. Pediatric Pulmonary and Cardiac Complications of Vertically Transmitted HIV Infection (P2C2 HIV) Study Group, National Heart, Lung, and Blood Institute. Pediatrics 1999;104:e14. 